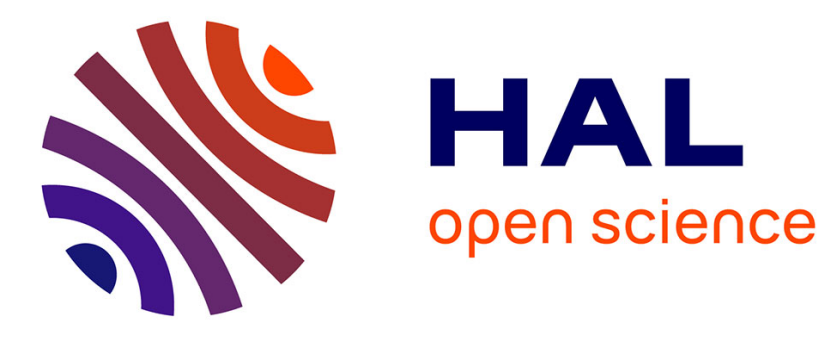

\title{
Les microARNs : en quoi intéressent-ils les cardiologues ? \\ Mathias Mericskay
}

\section{To cite this version:}

Mathias Mericskay. Les microARNs : en quoi intéressent-ils les cardiologues ?. La Presse medicale, 2015, 44 (7-8), pp.761-771. 10.1016/j.lpm.2015.05.005 . hal-01311546

\section{HAL Id: hal-01311546 \\ https: / hal.sorbonne-universite.fr/hal-01311546}

Submitted on 4 May 2016

HAL is a multi-disciplinary open access archive for the deposit and dissemination of scientific research documents, whether they are published or not. The documents may come from teaching and research institutions in France or abroad, or from public or private research centers.
L'archive ouverte pluridisciplinaire HAL, est destinée au dépôt et à la diffusion de documents scientifiques de niveau recherche, publiés ou non, émanant des établissements d'enseignement et de recherche français ou étrangers, des laboratoires publics ou privés. 
La Presse Médicale 2015: Journees Europeennes De La Societe Française De Cardiologie Dossier thématique : Les miARNs dans les pathologies cardiovasculaires Mathias Mericskay

\section{Les microARNs : en quoi intéressent-ils les cardiologues?}

Mathias Mericskay ${ }^{1,2}$

${ }^{1}$ INSERM, ERL U1164, Unité Biologie de 1'Adaptation et du Vieillissement, Paris F-75005, France, UPMC Université Paris 06

${ }^{2}$ CNRS, UMR8256, Unité Biologie de l'Adaptation et du Vieillissement, Institut de Biologie Paris-Seine (IBPS), Paris F-75005, France, Sorbonne Universités, UPMC Université Paris 06

\section{Correspondance :}

Mathias Mericskay, Equipe Génétique et Physiopathologie des Tissus Musculaires,

UMR8256 Unité Biologie de l'Adaptation et du Vieillissement, BP 256, 7 quai Saint Bernard, 75252 Paris Cedex 5, France.

Mathias.mericskay@upmc.fr 
La Presse Médicale 2015: Journees Europeennes De La Societe Française De Cardiologie

Dossier thématique : Les miARNs dans les pathologies cardiovasculaires Mathias Mericskay

\section{Points essentiels}

- Les miARNs sont des ARN de 21-22 nucléotides issus de gènes non codants ou d'introns de gène codant impliqués dans la répression des ARN messagers cellulaires par le complexe RISC.

- Près de 2000 miARNs sont identifiés dans le génome humain et des centaines d'entre eux voient leur expression augmentée ou diminuée en situation pathologique, modifiant de manière globale le taux d'expression des protéines cardiaques et vasculaires.

- Les miARNs affectent la plupart des processus cellulaires impliqués dans la physiopathologie cardiovasculaire : infarctus du myocarde, hypertrophie ventriculaire gauche, la fibrose cardiaque et troubles de la conduction.

- Les miARNs constituent un nouveau type de cible thérapeutique pouvant être introduits dans les cellules sous différentes formes, synthétique ou vecteur d'expression, ou bien inhibés par des oligonucléotides antisens appelés anti-miRs.

- Les miARNs sont excrétés activement par certaines cellules dont les fibroblastes cardiaques et les cellules endothéliales au cours de certains processus physiopathologiques, ou passivement lors de la mort cellulaire et peuvent être quantifiés dans tous les fluides corporels. Ils émergent donc comme une nouvelle catégorie de biomarqueurs des pathologies cardiovasculaires, notamment dans le contexte de l'infarctus du myocarde. 
La Presse Médicale 2015: Journees Europeennes De La Societe Française De Cardiologie

Dossier thématique : Les miARNs dans les pathologies cardiovasculaires Mathias Mericskay

MicroRNAs: what cardiologists should know about them?

\section{Key points}

- MiRNAs are small 21-22 nucleotides long RNAs transcribed from non coding genes or introns of coding genes that are involved the repression of cellular messenger RNAs by the RISC complex.

- Close to 2000 miRNAs have been identified in the human genome and the expression of hundreds of them is increased or decreased in pathological condition, modifying on a global scale the expression of cardiac and vascular proteins.

- MiRNAs regulate most cellular processes involved in the cardiovascular pathophysiology including apoptosis, proliferation and migration, left ventricle hypertrophy, cardiac fibrosis and conduction.

- MiRNAs form a new class of therapeutic targets that can be introduced in the cells in various forms (synthetic molecules, expression vectors) or inhibited by antisens oligonucleotides called anti-miRs.

- MiRNAs are actively secreted by specific cells including cardiac fibroblasts and endothelial cells during certain pathophysiological processes, or passively following cell death and can be quantified in all kind of body fluids. They emerge as a new category of cardiovascular disease biomarkers, notably in the context of myocardial infarction. 
La Presse Médicale 2015: Journees Europeennes De La Societe Française De Cardiologie Dossier thématique : Les miARNs dans les pathologies cardiovasculaires Mathias Mericskay

Abréviations : Ago, protéine argonaute ; CE, cellules endothéliales ; CML, cellules musculaire lisses ; DGCR8, Di George critical region 8 ; cTNT : cardiac Troponin T ; IGF1, insulin-like growth factor 1, MAPK, mitogen-activated protein kinase ; miR, microARN; miR*, brin opposé au miR dominant ; nt, nucléotides ; RISC, RNA-induced silencing complex ; STEMI , ST segment elevation myocardial infarction ; TRBP, TAR RNA binding protein 
La Presse Médicale 2015: Journees Europeennes De La Societe Française De Cardiologie Dossier thématique : Les miARNs dans les pathologies cardiovasculaires Mathias Mericskay

Découverts il y a une quinzaine d'années chez les mammifères, les miARNs (miARNs), sont des très petits ARN non codants de 21 à 22 nucléotides (nt) impliqués dans la modulation de l'expression des protéines cellulaires. Ils sont une des découvertes majeures de la dernière décennie, notamment dans le domaine de la recherche biomédicale. Les années 2000 auront vu une véritable explosion des publications scientifiques dans tous les domaines de la biologie avec un rythme de près de 500 publications par an rien que dans le domaine de la biologie cardiovasculaire ces 2 dernières années. Les miARNs ont émergé comme des acteurs majeurs des processus pathologiques affectant le système cardiovasculaire comme l'hypertrophie et la fibrose cardiaque, les maladies coronariennes, les arythmies ou l'athérosclérose [1]. Nous reprendrons ici de manière synthétique les mécanismes de base de production et d'action des miARNs et les données de la littérature concernant leur rôle et leur émergence comme un nouveau type de cible thérapeutique dans les pathologies cardiovasculaires. Enfin nous aborderons les recherches cliniques en cours visant à établir ces miARNs comme des biomarqueurs de certaines pathologies cardiaques.

\section{Origine cellulaire des miARNs et mode de fonctionnement}

\section{Biosynthèse}

Les miARNs dérivent soit d'ARN non codants dits pri-miARN, soit d'ARN primaires de gènes codant pour une protéine dont un intron contient une séquence précurseur appelée «mirtron» (Figure 1). Des séquences répétées inverses séparées de quelques nucléotides s'apparient pour former des structures secondaires appelées tiges-boucles par repliement de l'ARN simple brin sur lui-même (Figure 1). Un pri-miARN peut contenir plusieurs de ces tiges boucles précurseurs de miARN (pré-miARN) et dans ce cas, ces derniers sont dit en « cluster ». Ces tiges-boucles sont clivées par le complexe microprocesseur formé de Drosha 


\section{La Presse Médicale 2015: Journees Europeennes De La Societe Française De Cardiologie}

\section{Dossier thématique : Les miARNs dans les pathologies cardiovasculaires Mathias Mericskay}

et DGSCR8. Les mirtrons sont clivés par la machinerie d'épissage des introns ; coproduits avec les ARN messagers (ARNm), ils sont potentiellement impliqués dans des fonctions reliées à celles du gène hôte. Les pré-miARN (55-70 nt) sont exportés vers le cytoplasme où ils sont maturés par DICER en association avec TRBP. Ce complexe coupe la boucle et l'extrémité de la tige du pré-miARN et recrute une protéine de la famille Argonaute (Ago) qui déroule le duplex de miARN pour sélectionner un des 2 brins, généralement le brin avec une base A ou U en 5' qui permet un détachement plus facile du duplexe. Ce brin, dit dominant, est le miARN mature qui constitue avec une protéine Ago, le complexe RISC d'interférence par ARN. L'autre brin, dit passager (noté *), est en général dégradé mais peut parfois être aussi chargé dans un complexe RISC. La nomenclature actuelle des miARN est basée sur leur position en 5' (-5p, ex : miR-1-5p) ou 3' (-3p) du précurseur tige-boucle (voire exemple de miR-378, Figure 2).

\section{Répression des ARN messagers par le complexe RISC}

Le principal mode d'action des miARN est la répression des ARNm codant les protéines. Au sein du complexe RISC, le miARN s'hybride à des séquences complémentaires inverses dans l'ARNm, avec un rôle prépondérant de la séquence en 5' du miARN comprise entre les nucléotides 2 et 7-8, appelée séquence « graine » dont l'ancrage peut suffire à déclencher un effet répresseur, bien qu'il soit renforcé par des interactions supplémentaires en 3'. Ceci confère mathématiquement un nombre élevé de cibles potentielles. Cependant il faut tenir compte du fait que la structure secondaire de l'ARNm cible joue un rôle prépondérant pour l'accessibilité du miARN et que les deux doivent être présents dans la même cellule pour interagir, ces 2 paramètres réduisant fortement la combinatoire entre miARN et ARNm cibles Néanmoins un miARN peut contrôler des dizaines, voire des centaines d'ARNm et un même ARNm peut être ciblé de manière convergente par plusieurs miARNs. 
La Presse Médicale 2015: Journees Europeennes De La Societe Française De Cardiologie Dossier thématique : Les miARNs dans les pathologies cardiovasculaires Mathias Mericskay

Les sites de fixation des miARN dans les ARNm sont retrouvés à part égale entre les exons et la partie 3' non traduite. L'interaction avec la partie 3' nt semble cependant avoir l'effet le plus déterminant. Plusieurs modes d'actions du complexe RISC ont été décrits et des revues détaillées sur ces mécanismes moléculaires sont déjà disponibles [2]. On distingue principalement deux modes d'actions ; l'un basé sur la destruction de l'ARNm cible suite à l'interaction avec le complexe RISC ; l'autre n'affectant pas quantitativement l'ARNm cible mais bloquant sa traduction en protéine. Les méthodes d'identification des cibles des miARNs sont en constant progrès et combinent des approches bioinformatiques avec des criblages parallèles des niveaux d'expression des $\mathrm{ARNm}$ et des protéines ainsi que des techniques d'immunoprécipitation des duplex miARN-ARNm que nous ne détaillerons pas ici. Les miARNs sont donc des répresseurs globaux de l'expression protéique mais leur effet sur les ARNm ciblés n'est pas saturant et la répression sur le niveau d'expression des protéines est le plus souvent d'un facteur inférieur à 2. Notons que lorsque le miARN réprime l'expression d'une protéine dont l'action est elle-même inhibitrice d'une fonction (exemple : répresseur transcriptionnel), l'effet final est une activation de cette fonction. Enfin, il faut noter que si le principal mode d'action des miARN, décrits à ce jour, est intracellulaire, c'est à dire que la cellule produit les miARNs régulateurs de ses propres ARNm, une fonction paracrine est en train d'émerger. Plusieurs études montrent que les miARN peuvent être excrétés par différentes voies, sécrétion passive lors de la nécrose cellulaire, ou production active de différents types de vésicules. Ces miARN «paracrines » peuvent dans certains modèles expérimentaux pénétrer dans les cellules adjacentes et réprimer certains de leurs ARNm et modifier leur phénotype [1].

\section{Données de la recherche sur le rôle des miARNs dans le système cardiovasculaire}




\section{La Presse Médicale 2015: Journees Europeennes De La Societe Française De Cardiologie}

\section{Dossier thématique : Les miARNs dans les pathologies cardiovasculaires Mathias Mericskay}

Les miARNs ont été impliqués dans la différenciation des cellules cardiaques et vasculaires et dans pratiquement toutes les formes de maladies cardiovasculaires à ce jour. Les études sur les modèles souris de remodelage cardiaque et vasculaire pathologique (hypertrophie par surcharge en pression, infarctus du myocarde) ont montré que de nombreux miARNs voient leur profil d'expression fortement réprimé ou activé dans ce contexte (Figure 3).

\section{Rôle des miARN cardiaques dans les modèles souris de pathologies cardiaques}

L'hypertrophie du ventricule gauche (HVG) est l'un des modèles de pathologie cardiaque dans lequel le rôle des miARNs a été le plus extensivement exploré. Les miARNs de type miR-1, miR-133 et miR-378 dont l'expression est forte dans le myocarde à l'état physiologique, voient leur expression diminuée dans des modèles de HVG chez la souris induits par constriction de l'aorte ou par administration d'agonistes adrénergiques [3,4]. Ces miARNs ont été impliquées dans la répression d'ARNm codant des protéines de signalisation impliquées dans le processus d'hypertrophie comme les voies MAPK, les petites protéines G de la famille RhoA, la voie calcineurine/NFAT et la voie insuline/IGF1. MiR-378 est un mirtron localisé dans l'intron 1 du gène codant le cofacteur transcriptionnel PGC1 $\beta$ impliqué dans la biogénèse mitochondriale et la $\beta$-oxydation des acides gras. Notre équipe a montré que miR-378 et son brin opposé miR-378* répriment des protéines du métabolisme énergétique et du cytosquelette impliqués dans ces processus de remodelage cardiaque [5]. Les miR-199b, miR-132 et miR-212, augmentés dans le cœur insuffisant chez l'homme et la souris, répriment des voies de signalisation anti-hypertrophique et pro-autophagique [6,7]. Le cas des mirtrons miR-208a et -208b et 499 issus d'introns de gène codant pour des chaines lourde de myosine (CLM) est plus complexe. MiR-208a est coproduit avec le gène Myh6 codant la CLM rapide $\alpha$ et voit son expression diminuée dans l'HVG chez la souris et chez l'homme [8]. Cependant l'inactivation complète de miR-208a chez la souris aboutit à la 


\section{La Presse Médicale 2015: Journees Europeennes De La Societe Française De Cardiologie}

\section{Dossier thématique : Les miARNs dans les pathologies cardiovasculaires Mathias Mericskay}

surexpression de Thrap1, un répresseur transcriptionnel associé au récepteur à l'hormone thyroïdienne qui bloque l'induction caractéristique dans le cœur hypertrophique, des gènes Myh7 (CLM lente $\beta$ ) et Myh7b et des mirtrons associés miR-208b et miR-499. , très abondant dans le cœur et dont nous verrons l'émergence comme biomarqueur de l'infarctus du myocarde (voir section biomarqueur).

Un des processus important dans lequel les miARNs sont impliqués est la fibrose cardiaque dont on connaît les effets délétères sur la biomécanique cardiaque et la conduction du signal de dépolarisation. De nombreux miARNs, dont miR-21 et miR-29 sont parmi les mieux caractérisés, jouent un rôle dans la prolifération des fibroblastes cardiaques et la synthèse excessive de collagène dans le cœur malade [9]. De manière intéressante miR-21* est retrouvé dans des vésicules de sécrétion produites par les fibroblastes cardiaques et semble agir comme une molécule paracrine induisant l'hypertrophie des cardiomyocytes adjacents [10].

L'infarctus du myocarde constitue l'une des pathologies dans laquelle les changements d'expression des miARNs sont majeurs [11]. L'un des points clé de la pathogénèse de l'infarctus est la mort des cardiomyocytes. Alors que certains miARNs protecteurs des cardiomyocytes comme miR-24 sont réprimés, Les miARNs de la famille miR-15 et miR-34 ainsi que miR-1 sont surexprimés et répriment des protéines impliqués dans la survie cellulaire, le maintien de l'intégrité mitochondriale et la conduction cardiaque. MiR-214 et miR-132 sont activés au cours de l'infarctus du myocarde mais semblent au contraire des miR-15, -34 et -1 jouer un rôle protecteur en limitant l'étendue de la lésion ischémique [11]. Ces deux miARNs répriment l'ARNm codant l'échangeur $\mathrm{Na}^{+} / \mathrm{Ca}^{2+} \mathrm{NCX} 1$ qui, en situation d'infarctus, peut fonctionner en mode inverse et contribuer à augmenter la charge calcique des 


\section{La Presse Médicale 2015: Journees Europeennes De La Societe Française De Cardiologie}

\section{Dossier thématique : Les miARNs dans les pathologies cardiovasculaires Mathias Mericskay}

cardiomyocytes. MiR-214 réprime aussi l'expression de protéines impliquées dans l'activation du pore de transition de perméabilité mitochondrial comme la cyclophilline D.

La régulation de l'expression des protéines impliquées dans la régulation des flux calciques et des canaux ioniques impliqués dans la génération du potentiel d'action constitue un champ important de l'action des miARNs dans le myocarde. Le niveau d'expression de l'échangeur NCX1 semble notamment être l'objet de régulations par de multiples miARNs puisque outre sa répression par miR-214 et miR-132 mentionnés ci-dessus, notre équipe a montré que miR1 réprime aussi son expression et que la chute de miR-1 observée dans un contexte d'insuffisance cardiaque liée à une cardiomyopathie dilatée non ischémique [12], conduit à une surexpression anormale de cet échangeur qui peut perturber l'homéostasie calcique du cardiomyocyte. Nous avons montré cependant qu'une autre cible de miR-1, l'annexine A5 dont l'expression augmente également dans le cœur insuffisant, et qui est connue pour fixer le $\mathrm{Ca}^{2+}$ et interagir avec NCX1, ralentit son activité d'extrusion du $\mathrm{Ca}^{2+}$ [12]. L'hyperphosphorylation du récepteur à la ryanodine RyR dans le myocyte défaillant est une cause majeure de relargage anormal de $\mathrm{Ca}^{2+}$ en diastole, un processus hautement arythmogène. Les miR1- et -133 sont augmentés dans un modèle de tachycardie auriculaire droite chez le chien et miR-133 réprime l'ARNm codant les sous-unités catalytique de la phosphatase 2A conduisant à une hyperphosphorylation du RyR [13]. Dans le même type de modèle ou chez des patients atteints de rhumatisme cardiaque, le miR-328 est induit et réprime les sous-unités de canaux $\mathrm{Ca}^{2+}$ de type $\mathrm{L}$ contribuant à raccourcir le potentiel d'action et la force de la contraction atriale [14]. 


\section{La Presse Médicale 2015: Journees Europeennes De La Societe Française De Cardiologie}

\section{Dossier thématique : Les miARNs dans les pathologies cardiovasculaires Mathias Mericskay}

L'athérosclérose et l'hypertension artérielle constituent des facteurs de risques majeurs pour les maladies coronariennes, l'infarctus du myocarde et l'HVG respectivement. Les cellules musculaires lisses (CML) et endothéliales (CE) expriment des groupes spécifiques de miARN. Les lésions athéromateuses sont induites dans des zones sensibles de bifurcation des vaisseaux par la combinaison de facteurs associant altération locale des contraintes de cisaillement affectant la perméabilité et la prolifération des CE, et l'accumulation de macrophages spumeux chargés en lipoprotéines et cholestérol contribuant à l'inflammation et à la formation d'un cœur nécrotique déstabilisant la plaque. Certains miARNs comme miR10a jouent un rôle protecteur en réprimant l'activation de la voie NF-kB liée à l'inflammation [15]. A l'inverse une étude du groupe de Tedgui (Paris) a montré que miR-92a est surexprimé dans les $\mathrm{CE}$ des zones de lésions athéromateuses où il réprime Klf2, un répresseur de l'inflammation et de la prolifération des CE (ces processus sont donc activés) [16]. MiR-24 augmente également dans les $\mathrm{CE}$ après infarctus et à l'inverse de son action dans les cardiomyocytes, induit la mort des $\mathrm{CE}$, ce qui rend son rôle particulièrement ambigu dans cette maladie [11]. L'endothélium des zones pro-athéromateuses est plus perméable aux lipoprotéines de basse densité (LDL) transportant le cholestérol. Ces LDL sont sujettes à l'oxydation et pénètrent l'intima artérielle où elles contribuent à activer les macrophages. Le miARN let-7g est un répresseur de l'ARNm codant le récepteur au LDL oxydé LOX1 et inversement l'activation de LOX1 par son ligand tend à réprimer l'expression de let-7g [17]. Cette étude a aussi montré que le niveau de let-7g est réduit dans le sérum de patients hypercholestérolémiques. Enfin une étude montre que miR-126-5p, un miARN abondant dans les CE est excrété via des microvésicules lors de l'apoptose des CE dans l'athérosclérose. Lorsqu'il est capturé par les $\mathrm{CE}$ adjacentes, il inhibe un répresseur de la voie chemokine CXCL12-CXCR4 qui se retrouve par conséquent dé-réprimée et enclenche le processus de 
La Presse Médicale 2015: Journees Europeennes De La Societe Française De Cardiologie

Dossier thématique : Les miARNs dans les pathologies cardiovasculaires Mathias Mericskay

migration et de prolifération des CE adjacentes permettant de réparer l'endothélium vasculaire [18].

Les CML de la média artérielle expriment également des miARN. De nombreux autres miARNs (miR-143, miR-145, miR-27a/b, miR-155, miR-421, miR-483-3p) sont impliqués dans la répression d'ARNm codant différentes protéines du système RAA et apeline qui est suractivé dans l'hypertension essentielle [19].

Ces études et bien d'autres que nous n'avons pas pu citer, convergent pour montrer que les modulations de l'expression de certains miARN jouent un rôle important dans la réponse à un stress cellulaire et dans la pathogénèse des maladies cardiovasculaires. Une limitation actuelle de ces études précliniques est que les mécanismes moléculaires proposés sont basés sur des approches réductionnistes quelque peu simplificatrices visant à expliquer le mécanisme d'action d'un microARNmiARN donné par son action sur un nombre limité de cibles, en général choisies par les auteurs parmi des centaines de candidats possibles sur la base de leur implication plausible dans la pathologie. Cette approche candidat est due à la lourdeur des techniques d'identification et de validation des cibles qui ne sont pas toutes applicables aux études in vivo et nécessitent de passer par des modélisations in vitro pour être validées. Ces cibles sont certainement importantes mais les futures recherches dans le domaine devront employer des approches moins biaisées pour identifier l'ensemble des cibles contrôlées par un miARN donné dans une situation pathologique déterminée. Le développement des techniques d'immunoprécipitation des complexes RISC associées aux techniques de séquençage des duplex miARN-ARNm devraient permettre à l'avenir d'aborder de manière plus globale et plus exhaustive la question des cibles pathologiques des miARNs. 
La Presse Médicale 2015: Journees Europeennes De La Societe Française De Cardiologie Dossier thématique : Les miARNs dans les pathologies cardiovasculaires Mathias Mericskay

Enfin, notons que plusieurs études ont cherché à comparer les profils d'expression des miARNs dans le myocarde humain entre les états physiologiques et pathologiques. Ces études, basées sur des techniques de séquençage à haut débit montrent que plusieurs centaines de miARNs sont détectées dans le cœur humain mais il est frappant de constater que les données publiées concernant le profil d'expression individuel de chaque miARN peuvent diverger de manière importante et parfois même contradictoire [20,21]. Evidemment, comme toutes les études de ce type, les données sont obtenues à partir de biopsies de cœurs prélevés en phase terminale de l'insuffisance cardiaque terminale, au moment de la transplantation ou de la pose d'une assistance ventriculaire, sur des patients avec des étiologies diverses et en nombre restreint, tous ces paramètres pouvant en partie expliquer ces différences.

\section{Les miARNs comme cible thérapeutique}

\section{Rationnel et méthodologie}

Le grand nombre d'études montrant l'impact de la dérégulation des miARNs dans les pathologies cardiovasculaires conduit à penser que ces derniers pourraient constituer des cibles thérapeutiques de choix. L'objectif thérapeutique est soit de restaurer un miARN pour lequel la diminution observée de l'expression serait pathogénique (et non adaptative) ou inversement d'inhiber un miARN anormalement surexprimé.

La nature chimique très conservée entre tous les miARNs a permis de développer rapidement une chimie de synthèse efficace pour générer soit des précurseurs de miARNs, appelés « miR mimes » pour augmenter un miARN, soit des oligonucléotides antisens d'un miARN donné appelés « anti-miR» ou « antagomir ». Cette maîtrise de la chimie sous-jacente au design de la molécule thérapeutique permet une approche «candidat» qui est plus facile à mettre en œuvre que pour les médicaments classiques conçus pour cibler des protéines spécifiques, car 


\section{La Presse Médicale 2015: Journees Europeennes De La Societe Française De Cardiologie}

\section{Dossier thématique : Les miARNs dans les pathologies cardiovasculaires Mathias Mericskay}

ces dernières ont des conformations et des propriétés biochimiques bien plus différentes les unes des autres (code à 20 acides aminés) que les ARNs (4 bases).

La taille relativement réduite des précurseurs miR mimes (50 à 70 bp comme les précurseurs endogènes) permet de les vectoriser dans des lipoparticules ou éventuellement dans des vecteurs viraux comme le AAV9 qui a un tropisme renforcé pour les cellules cardiaques. Encore plus petits et d'utilisation plus aisée, les anti-miRs sont des oligonucléotides stabilisés de 20 à 22 nucléotides qui peuvent être injectés par voie sous-cutanée ou intraveineuse (Figure 4).

Le deuxième argument du ciblage thérapeutique des miARNs est leur mode d'action ciblant l'expression de dizaine de protéines cellulaires supposées agir dans des processus biologiques convergents impactant sur la pathologie. Ceci est parfois présenté comme un changement de paradigme par rapport aux molécules pharmaceutiques classiques qui cibleraient un récepteur donné de manière très spécifique mais cet argument ne semble pas correct, car d'une part la spécificité d'action «mono-cible» des molécules pharmaceutiques actuellement sur le marché est souvent loin d'être démontrée et de toute façon, des récepteurs comme les récepteurs $\beta$-adrénergiques ciblés par les $\beta$-bloquants contrôlant des dizaines de protéines cellulaires au niveau du cytosol mais aussi des voies de signalisation agissant dans le noyau sur l'expression de centaines de gènes dans les cardiomyocytes. Il n'y a donc pas de différence conceptuelle majeure dans le ciblage des miARN par rapport à la thérapeutique actuelle, notamment dans la difficulté de prédire les effets secondaires indésirables.

Une des complications propres aux miARNs vient de leur action croisée sur des cibles communes qui fait penser que surexprimer ou inhiber un seul miARN n'aura pas l'effet quantitatif souhaité sur certains de ses ARNm cibles car ces derniers peuvent être réprimés 


\section{La Presse Médicale 2015: Journees Europeennes De La Societe Française De Cardiologie}

\section{Dossier thématique : Les miARNs dans les pathologies cardiovasculaires Mathias Mericskay}

par d'autres miARNs toujours présents dans la cellule. Il existe des stratégies alternatives pour contrer ce problème comme l'utilisation soit d'inhibiteurs LNA courts de 6 à 7 nucléotides qui peuvent s'hybrider avec la séquence graine commune à un ensemble de miARNs d'une même famille (voir section biogénèse et mode d'action). Le risque étant cependant avec ces inhibiteurs courts qu'ils soient très peu spécifiques et capables de s'hybrider à de nombreux autres miARNs en dehors de la séquence graine ou directement à des ARN cellulaires de toute nature avec des conséquences inconnues sur le fonctionnement cellulaire. Pour cibler un ensemble de miARNs, même non reliés entre eux sur le plan de la séquence nucléotidique, une autre approche a été conçue basée sur la création de vecteur viraux permettant l'expression d'un ARN « éponge », c'est à dire un ARN artificiel dont la seule fonction est de contenir plusieurs copies d'une séquence cible d'un ou plusieurs miARN pour les capturer et alléger leur effet répresseurs sur les ARNm cellulaires.

\section{Quelques exemples de thérapie miARN}

Depuis la publication des résultats du premier essai clinique de phase 2 basé sur l'utilisation d'un LNA anti-miR-122 (Miravisen, Santaris Pharma) pour le traitement de l'hépatite C chronique [22], ou l'annonce d'un essai de phase I de traitement du cancer du foie par un miR-mime encapsulé dans des liposomes permettant de surexprimer le miARN miR-34 aux propriétés suppresseur de tumeur (MRX34 développé par Mirna Therapeutics, https://www.clinicaltrials.gov; identifier: NCT01829971), la thérapie par modulation des miARNs n'est plus réservé au domaine de la science fiction. En cardiologie, les études précliniques sur les modèles rongeurs citées précédemment montrent qu'un certain nombre d'anti-miR ciblant les miR-133, -208a, -378, -199b, peuvent fortement limiter le développement d'une HVG et d'une fibrose dans des modèles expérimentaux de constriction de l'aorte ascendante ou d'administration d'agonistes pro-hypertrophiques [3,4,6,8]. L'anti- 
La Presse Médicale 2015: Journees Europeennes De La Societe Française De Cardiologie Dossier thématique : Les miARNs dans les pathologies cardiovasculaires Mathias Mericskay

miR ciblant miR-1 a des propriétés antiarythmique [23]. Cependant il faut noter que, comme la plupart des médicaments, les anti-miRs injectés par voie intraveineuse ou sous-cutanée s'accumulent massivement dans le foie et les reins, nécessitant des doses substantielles pour atteindre les cellules du système cardiovasculaire et suscitant des questions sur la toxicité potentielle des doses utiles.

Ces études sont intéressantes sur le plan de la preuve de concept mais il faut reconnaitre que les modèles d'HVG chez la souris restent assez éloignés de la clinique humaine. Il est donc intéressant de noter que certaines équipes commencent à transférer ces approches sur des modèles dits de «gros animaux» qui ont l'avantage, outre d'être plus proches de la physiologie humaine, de permettre de développer des stratégies d'adressage ciblé des antimiRs. Il a ainsi été montré que l'injection en intracoronaire d'un anti-miR-92a encapsulé dans des microsphères biodégradables stimule l'angiogenèse et limite le remodelage sur un modèle miniporc (25 kg) d'infarctus aigu reperfusé [24].

\section{Les miARNs comme biomarqueurs des maladies cardiovasculaires}

La découverte progressive de la présence des miARNs dans tous les fluides corporels (plasma, urine, salive, liquide cérébrospinal) a suscité un intérêt croissant pour leur potentiel comme biomarqueurs. Les miARNs extracellulaires sont retrouvés dans des microparticules circulantes de type exosomes ou corps multivésiculaires qui pouraient correspondre à des sécrétions actives ou des particules apoptotiques dérivées de cellules mortes, en association avec des protéines fixant l'ARN ou en complexe avec le cholestérol-HDL. Ces miARNs apparaissent comme étant relativement stables dans ces fluides mais il faut noter que les conditions de prélèvement, l'heure du prélèvement, la prise de nourriture et le choix de l'anticoagulant utilisé lors du prélèvement peuvent affecter le résultat [25]. D'autre part, les 


\section{La Presse Médicale 2015: Journees Europeennes De La Societe Française De Cardiologie}

\section{Dossier thématique : Les miARNs dans les pathologies cardiovasculaires Mathias Mericskay}

méthodes de détection utilisées, PCR en temps réel, hybridation sur micropuces ou séquençage ARN de nouvelle génération, ont des sensibilités différentes. La méthode par PCR en temps réel est sans doute la plus employée à ce jour car très sensible et facile à mettre en œuvre mais elle présente de réelles difficultés de normalisation. Ces différences dans les différents paramètres d'analyse, qui ne sont pas anormales pour un champ de recherche encore jeune, rendent compliquée la comparaison des différentes études cliniques parue à ce jour. Malgré tout certains miARNs émergent comme ayant un fort potentiel de biomarqueur. Il ne sera pas possible dans cette mise au point de les mentionner (voire [25] pour une revue détaillée). Nous citerons principalement les études ayant inclus plusieurs centaines d'individus qui ont atteint une puissance statistique correcte.

\section{Infarctus aigu du myocarde}

Les miARNs abondant dans le myocarde tels que miR-1, miR-133a/b, miR-499 et miR208a/b sont retrouvés comme fortement augmentés dans le sérum ou le plasma de patients en phase aigue d'infarctus du myocarde (IAM) [25]. Dans une étude sur 407 patients se présentant avec des symptomes de syndrome coronarien aigu, les miR-208b et $-499-5 p$ (normalisés sur miR-17) discriminaient les patients en IAM des patients non IAM (aire sous la courbe $\mathrm{ASC}=0,82$ et 0,79 , respectivement $)$ mais moins bien que la $\mathrm{cTnT}(\mathrm{ASC}=0,95)[26]$. La précision du diagnostic était nettement augmentée pour les miARNs si les auteurs considéraient seulement les échantillons prélevés dans les 24 heures après admission suggérant que la stabilité de ces miARNs dans la circulation après relarguage par les cardimyocytes nécrotiques est limitante. Les 2 miARNs avaient également une valeur pronostic comparable à la cTnT pour la prédiction d'un événement cardiovasculaire adverse dans les 30 jours mais n'apportaient pas d'incrément en combinaison avec la cTnT. Une autre étude a montré que dans le cas des patients gériatriques pour lesquels il est difficile de 
La Presse Médicale 2015: Journees Europeennes De La Societe Française De Cardiologie Dossier thématique : Les miARNs dans les pathologies cardiovasculaires Mathias Mericskay distinguer un IAM-NSTEMI d'une insuffisance cardiaque aigüe sans IAM, le niveau d'élévation du miR-499-5p (normalisé sur miR-17) permettait de mieux distinguer ces deux types de patients $(\mathrm{AUC}=0,86)$ dans les sous-groupes présentant des élévations modestes de $\mathrm{cTnT}(\mathrm{cTnT} \mathrm{AUC}=0,68$ et hs-cTnT $\mathrm{AUC}=0,70)[27]$. La valeur pronostic des biomarqueurs miARN pour l'évolution de la maladie reste sujette à caution. Une étude longitudinale sur 12 mois des équipes de Bauters et Pinet (Lille) et Thum (Hanovre) n'a pas montré d'association entre les niveaux de miR-133a et -423-5p avec les indices de fonction et remodelage VG [28].

\section{Maladies coronariennes, angor stable et instable}

Les études sur les patients atteints de maladie coronarienne sont plus confuses dans le sens où beaucoup d'entre elles reposent sur des petits échantillonnages dont la signification clinique n'est pas toujours évidente et peu d'entre elles reproduisent les résultats. L'augmentation des miR-155 associée au processus inflammatoire et miR-146a est retrouvée dans quelques études mais n'émergent pas clairement comme étant discriminants entre IAM et non IAM [25,29]. De même à ce stade les tentatives de définir des groupes de miARNs comme formant une signature permettant de distinguer les patients en angor stable des patients en angor instable n'apparaissent pas comme très concluantes avec peu de reproductibilité entre les études. De manière plus intéressante, dans une étude sur la cohorte de Bruneck de 1995 à 2005 (n=820), qualifiée de prospective dans le titre mais qui était en fait plutôt rétrospective car les miARNs ont été mesurés après la fin de l'étude dans les échantillons de 1995, le niveau de miR-126 dans les cellules mononuclées du sang périphérique est associé positivement, et ceux de miR223 et miR-197 négativement, avec la survenue d'un IM dans les 10 ans [30]. L'addition des 3 miARNs au score de risque de Framingham pour les maladies coronariennes donnait un index net de reclassification (NRI) de $16,86 \%$ (IC : $-1,99 \%$ à $35,71 \% ; p=0,080)$. Ces données nécessiteraient d'être confirmées dans une cohorte indépendante mais suggèrent que 
La Presse Médicale 2015: Journees Europeennes De La Societe Française De Cardiologie Dossier thématique : Les miARNs dans les pathologies cardiovasculaires Mathias Mericskay

la quantification des miARNs circulants pourrait avoir une valeur pronostic pour les maladies coronariennes.

\section{Conclusion}

En l'espace de quelques années, un effort intensif de recherche au niveau international a permis d'apporter une masse impressionnante de données sur le rôle des miARNs dans les pathologies cardiovasculaires. De nombreuses questions restent évidemment en attente, notamment une meilleure compréhension des règles stœchiométriques gouvernant les interactions entre miARN et ARNm est nécessaire ainsi que la prise de conscience que les études basées sur la caractérisation de quelques cibles candidates d'un miARN donné n'ont plus de sens au vu de leurs effets à grande échelle sur le protéome. Néanmoins les études précliniques convergent dans leur démonstration que la modulation de l'activité des miARNs peut avoir un impact sur les pathologies cardiovasculaires. Comme pour les autres cibles médicamenteuses, le problème des miARNs impliqués dans de multiples processus pathologiques dont le cancer soulève des questions quant aux méthodes de ciblage de l'organe malade, le domaine de la cardiologie interventionnelle étant cependant suffisamment à la pointe dans ce domaine pour donner des raisons d'espérer. Enfin nous en sommes encore aux prémices de l'utilisation des miARNs circulants comme biomarqueurs et leur gain en terme de spécificité reste encore largement à démontrer. Néanmoins, ces molécules de miARNs étant chimiquement toutes de nature quasi identique, cette propriété permet un développement technologique extrêmement rapide des méthodes de quantification et les miARNs pourraient un jour l'emporter sur un plan économique par rapport aux test ELISA basés sur des anticorps de type cTnT. Enfin, il faut noter que les microARN ne constituent que la partie émergée la mieux caractérisée du monde des $\mathrm{ARN}$ non codant dont on découvre ces dernières années l'étendue insoupçonnée [1]. Parmi eux, les ARN non codant longs (>200nt) sont impliqués 
La Presse Médicale 2015: Journees Europeennes De La Societe Française De Cardiologie Dossier thématique : Les miARNs dans les pathologies cardiovasculaires Mathias Mericskay

dans de nombreuses fonctions de régulation transcriptionnelle et post-transcriptionelle des gènes codants et émergent comme de nouveaux acteurs et biomarqueurs potentiels des pathologies cardiovasculaires.

Remerciements. Je remercie l'INSERM, le CNRS, l'UPMC et l'ANR pour le soutien aux recherches de l'équipe. Je remercie N. Chevallier-Mericskay (EFS, Créteil), J. Blanc et J-F. Decaux (UMR8256, UPMC Univ Paris 6) pour la lecture critique du manuscrit.

\section{Conflits d'intérêt : aucun}

\section{References}

[1] Thum T, Condorelli G. Long noncoding RNAs and micromoRNAs in cardiovascular pathophysiology. Circ Res 2015;116:751-62.

[2] Brodersen P, Voinnet O. Revisiting the principles of micromoRNA target recognition and mode of action. Nat Rev Mol Cell Biol 2009;10:141-8.

[3] Care A, Catalucci D, Felicetti F, Bonci D, Addario A, Gallo P, et al. MicroMoRNA133 controls cardiac hypertrophy. Nat Med 2007;13:613-8.

[4] Ganesan J, Ramanujam D, Sassi Y, Ahles A, Jentzsch C, Werfel S, et al. MiR-378 controls cardiac hypertrophy by combined repression of mitogen-activated protein kinase pathway factors. Circulation 2013;127:2097-106.

[5] Mallat Y, Tritsch E, Ladouce R, Winter DL, Friguet B, Li Z, et al. Proteome modulation in H9c2 cardiac cells by micromoRNAs miR-378 and miR-378. Mol Cell Proteomics MCP 2014;13.

[6] Da Costa Martins PA, Salic K, Gladka MM, Armand A-S, Leptidis S, el Azzouzi H, et al. MicroRNA-199b targets the nuclear kinase Dyrk1a in an auto-amplification loop promoting calcineurin/NFAT signalling. Nat Cell Biol 2010;12:1220-7. 
La Presse Médicale 2015: Journees Europeennes De La Societe Française De Cardiologie Dossier thématique : Les miARNs dans les pathologies cardiovasculaires Mathias Mericskay

[7] Ucar A, Gupta SK, Fiedler J, Erikci E, Kardasinski M, Batkai S, et al. The miRNA212/132 family regulates both cardiac hypertrophy and cardiomyocyte autophagy. Nat Commun 2012;3.

[8] Montgomery RL, Hullinger TG, Semus HM, Dickinson BA, Seto AG, Lynch JM, et al. Therapeutic inhibition of miR-208a improves cardiac function and survival during heart failure. Circulation 2011;124:1537-47.

[9] Viereck J, Bang C, Foinquinos A, Thum T. Regulatory RNAs and paracrine networks in the heart. Cardiovasc Res 2014;102;290-301

[10] Bang C, Batkai S, Dangwal S, Gupta SK, Foinquinos A, Holzmann A, et al. Cardiac fibroblast-derived microRNA passenger strand-enriched exosomes mediate cardiomyocyte hypertrophy. J Clin Invest 2014;124:2136-46.

[11] Boon RA, Dimmeler S. MicroMoRNAs in myocardial infarction. Nat Rev Cardiol $2015 ; 12: 135-42$.

[12] Tritsch E, Mallat Y, Lefebvre F, Diguet N, Escoubet B, Blanc J, et al. An SRF/miR-1 axis regulates NCX1 and annexin A5 protein levels in the normal and failing heart. Cardiovasc Res 2013;98:372-80.

[13] Belevych AE, Sansom SE, Terentyeva R, Ho H-T, Nishijima Y, Martin MM, et al. MicroRNA-1 and -133 increase arrhythmogenesis in heart failure by dissociating phosphatase activity from RyR2 complex. PloS One 2011;6. e28324.

[14] Lu Y, Zhang Y, Wang N, Pan Z, Gao X, Zhang F, et al. MicroRNA-328 contributes to adverse electrical remodeling in atrial fibrillation. Circulation 2010;122:2378-87.

[15] Fang Y, Shi C, Manduchi E, Civelek M, Davies PF. MicroRNA-10a regulation of proinflammatory phenotype in athero-susceptible endothelium in vivo and in vitro. Proc Natl Acad Sci U S A 2010;107:13450-5.

[16] Loyer X, Potteaux S, Vion A-C, Guerin CL, Boulkroun S, Rautou P-E, et al. Inhibition of microRNA-92a prevents endothelial dysfunction and atherosclerosis in mice. Circ Res 2014;114:434-43. 
La Presse Médicale 2015: Journees Europeennes De La Societe Française De Cardiologie Dossier thématique : Les miARNs dans les pathologies cardiovasculaires Mathias Mericskay

[17] Chen K-C, Hsieh I-C, Hsi E, Wang Y-S, Dai C-Y, Chou W-W, et al. Negative feedback regulation between microRNA let-7g and the oxLDL receptor. J Cell Sci 2011;124:4115-24.

[18] Zernecke A, Bidzhekov K, Noels H, Shagdarsuren E, Gan L, Denecke B, et al. Delivery of microRNA-126 by apoptotic bodies induces CXCL12-dependent vascular protection. Sci Signal 2009;2(100):ra81

[19] Chen L-J, Xu R, Yu H-M, Chang Q, Zhong J-C. The ACE2/Apelin Signaling, MicroRNAs, and Hypertension. Int J Hypertens 2015;2015:896861.

[20] Akat KM, Moore-McGriff D, Morozov P, Brown M, Gogakos T, Correa Da Rosa J, et al. Comparative RNA-sequencing analysis of myocardial and circulating small RNAs in human heart failure and their utility as biomarkers. Proc Natl Acad Sci U S A 2014;111:11151-6.

[21] Leptidis S, El Azzouzi H, Lok SI, de Weger R, Olieslagers S, Kisters N, et al. A deep sequencing approach to uncover the miRNOME in the human heart. PloS One 2013;8.e57800.

[22] Janssen HLA, Reesink HW, Lawitz EJ, Zeuzem S, Rodriguez-Torres M, Patel K, et al. Treatment of HCV infection by targeting microRNA. N Engl J Med 2013;368:1685-94.

[23] Yang B, Lin H, Xiao J, Lu Y, Luo X, Li B, et al. The muscle-specific microRNA miR1 regulates cardiac arrhythmogenic potential by targeting GJA1 and KCNJ2. Nat Med 2007;13:486-91.

[24] Bellera N, Barba I, Rodriguez-Sinovas A, Ferret E, Asin MA, Gonzalez-Alujas MT, et al. Single intracoronary injection of encapsulated antagomir-92a promotes angiogenesis and prevents adverse infarct remodeling. J Am Heart Assoc 2014; 3(5):e000946.

[25] Schulte C, Zeller T. microRNA-based diagnostics and therapy in cardiovascular disease-Summing up the facts. Cardiovasc Diagn Ther 2015;5:17-36 
La Presse Médicale 2015: Journees Europeennes De La Societe Française De Cardiologie Dossier thématique : Les miARNs dans les pathologies cardiovasculaires Mathias Mericskay

[26] Gidlof O, Smith JG, Miyazu K, Gilje P, Spencer A, Blomquist S, et al. Circulating cardio-enriched microRNAs are associated with long-term prognosis following myocardial infarction. BMC Cardiovasc Disord 2013;13. doi:10.1186/1471-2261-13-12.

[27] Olivieri F, Antonicelli R, Lorenzi M, D’Alessandra Y, Lazzarini R, Santini G, et al. Diagnostic potential of circulating miR-499-5p in elderly patients with acute non. Int J Cardiol 2013;167:531-6.

[28] Bauters C, Kumarswamy R, Holzmann A, Bretthauer J, Anker SD, Pinet F, et al. Circulating miR-133a and miR-423-5p fail as biomarkers for left ventricular remodeling after myocardial infarction. Int J Cardiol 2013;168:1837-40.

[29] Zhu J, Chen T, Yang L, Li Z, Wong MM, Zheng X, et al. Regulation of microRNA155 in atherosclerotic inflammatory responses by targeting MAP3K10. PloS One 2012;7(11):e46551

[30] Zampetaki A, Willeit P, Tilling L, Drozdov I, Prokopi M, Renard J-M, et al. Prospective study on circulating MicroRNAs and risk of myocardial infarction. J Am Coll Cardiol 2012;60:290-9. 
La Presse Médicale 2015: Journees Europeennes De La Societe Française De Cardiologie

Dossier thématique : Les miARNs dans les pathologies cardiovasculaires Mathias Mericskay

\section{Encadré 1 : les microARNs comme biomarqueurs}

\section{PRO}

- Grande stabilité dans le plasma

- Chimie identique pour tous les miARNs : plus simple et plus économique pour le développement technologique

- Méthode de détection par PCR très sensible

- Possibilité de combiner plusieurs miARNs pour augmenter la spécificité de l'information

\section{CONTRA}

- Pas de méthode de normalisation totalement satisfaisante

- Pas de valeur seuil de référence définie dans la population

- Niveaux dans le plasma affectés par la fonction rénale et la prise de certains médicaments

- Peu de miARNs strictement spécifiques d'un type cellulaire générant la question du tissu d'origine du biomarqueur et de sa signification physiopathologique 
La Presse Médicale 2015: Journees Europeennes De La Societe Française De Cardiologie Dossier thématique : Les miARNs dans les pathologies cardiovasculaires Mathias Mericskay

\section{Legendes}

Figure 1. Biosynthèse et modes d'action des microARNs. A. Les ARNs primaires non codant pri-miARN ou les intron de certains ARN messsagers formant les «mirtrons », génèrent des structures secondaires en tiges-boucles. Ces tiges-boucles sont clivées par le complexe microprocesseur Drosha/DGSCR8 ou par le complexe d'épissage pour former les pré-miARN. Ces derniers sont exportés vers le cytoplasme pour être clivés par le complexe DICER/TRBPC. Drosha et DICER sont des ARN endonucléase de type III et DGCR8 et TRBP des protéines d'ancrage aux structures ARN double brin. Le brin du miARN mature s'associe avec les protéines argonautes (Ago) pour former le complexe RISC qui reconnaît des séquences complémentaires dans les ARNm au niveau des exon ou de la séquence 3' non codante (NC). Lorsque le RISC contient la protéine Ago2, il peut cliver l'ARNm cible. Les autres Ago peuvent inhiber la traduction de l'ARNm en protéine qui nécessite le rapprochement des régions $3^{\prime}$ ' et $5^{\prime}$ pour le recrutement des ribosomes, et/ou induire sa déstabilisation via différents processus dont la dénadénylation en 3'. Les miARNs peuvent circuler entre les compartiments extra et intracelullaires. Les ARNm $(\approx 2000 \mathrm{nt})$ et les miARNs (21-22 nt) ne sont pas représentés à l'échelle.

\section{Figure 2. Exemple des mirtrons miR-378 et miR-378*}

A. ARN primaire du gène Ppargclb avec la structure en tige-boucle précurseur du miR dans l'intron 1. B. Séquence du pré-miR replié sur lui-même via l'appariement des bases complémentaires, avec la séquence du miR-378-5p en bleu et le miR-378-3p en rouge. C. Séquences des miRs matures produits par le complexe DICER/TRBP. Dans cet exemple, c'est le miR-378-3p qui contient une base A en 5' et qui est par conséquent sélectionné comme le brin dominant par la protéine Ago (noter que sa séquence est donnée dans l'ordre 5' $\rightarrow$ 3' du 
La Presse Médicale 2015: Journees Europeennes De La Societe Française De Cardiologie Dossier thématique : Les miARNs dans les pathologies cardiovasculaires Mathias Mericskay

pré-miR montré en B). Dans ce cas précis, miR-378-5p (*) peut également être détecté dans le cour mais reste moins abondant que miR-378.

\section{Figure 3. Implication des microARNARNs dans les pathologies cardiovasculaires}

Les modulations des miARNs indiquées sont considérées comme ayant un effet pathogène à quelques exceptions présentant des actions plus complexes: (1) l'augmentation de miR-214 dans l'infarctus limite la mort cellulaire, la baisse de miR-208a diminue l'hypertrophie, l'augmentation de miR-146a et miR-155 bloque la prolifération et l'hypertrophie (2) certains miARNs ont des actions opposées selon le type cellulaire: miR-24 a une action antiapoptotique dans les cardiomyocytes et pro-apoptotique dans les cellules endothéliales, miR21 a un effet bénéfique dans la réduction de l'infarctus du myocarde mais pro-fibrotique dans les modèles d'HVG; (3) miR-21* et miR-126-5p sont des miARNs excrétés par les fibroblastes ou les CE agissant de manière paracrine.

\section{Figure 4. Design des anti-miRs.}

Les oligonucléotides anti-miRs ont une séquence complémentaire inverse de celle du miARN ciblé avec lequel ils forment un hétéroduplex stable empêchant le miARN d'agir sur ses cibles ARNm. Les anti-miRs peuvent contenir des bases modifiées par la présence d'un groupe méthyl en position 2' du glucose et par un remplacement de la liaison phosphorodiester par une liaison phosphorothioate qui les rend résistants aux ARNases cellulaires. Un groupe cholestérol peut être ajouté en 3' de l'anti-miR pour favoriser sa pénétration dans les cellules. Les bases LNA contiennent un pont méthylène entre l'hydroxyle en position 2 et l'atome de carbone $4 \mathrm{du}$ glucose permettant de bloquer la conformation de la base et renforçant l'interaction avec la base opposée dans le miARNs. Les bases LNA permettent de générer des 
La Presse Médicale 2015: Journees Europeennes De La Societe Française De Cardiologie Dossier thématique : Les miARNs dans les pathologies cardiovasculaires Mathias Mericskay

anti-miRs court (comme montré en bas de la figure) mais sont également employées mixés avec des bases normales dans des anti-miRs plus longs de 15 à 20 nucléotides (non montré). 
La Presse Médicale 2015: Journees Europeennes De La Societe Française De Cardiologie Dossier thématique : Les miARNs dans les pathologies cardiovasculaires Mathias Mericskay

Figure 1

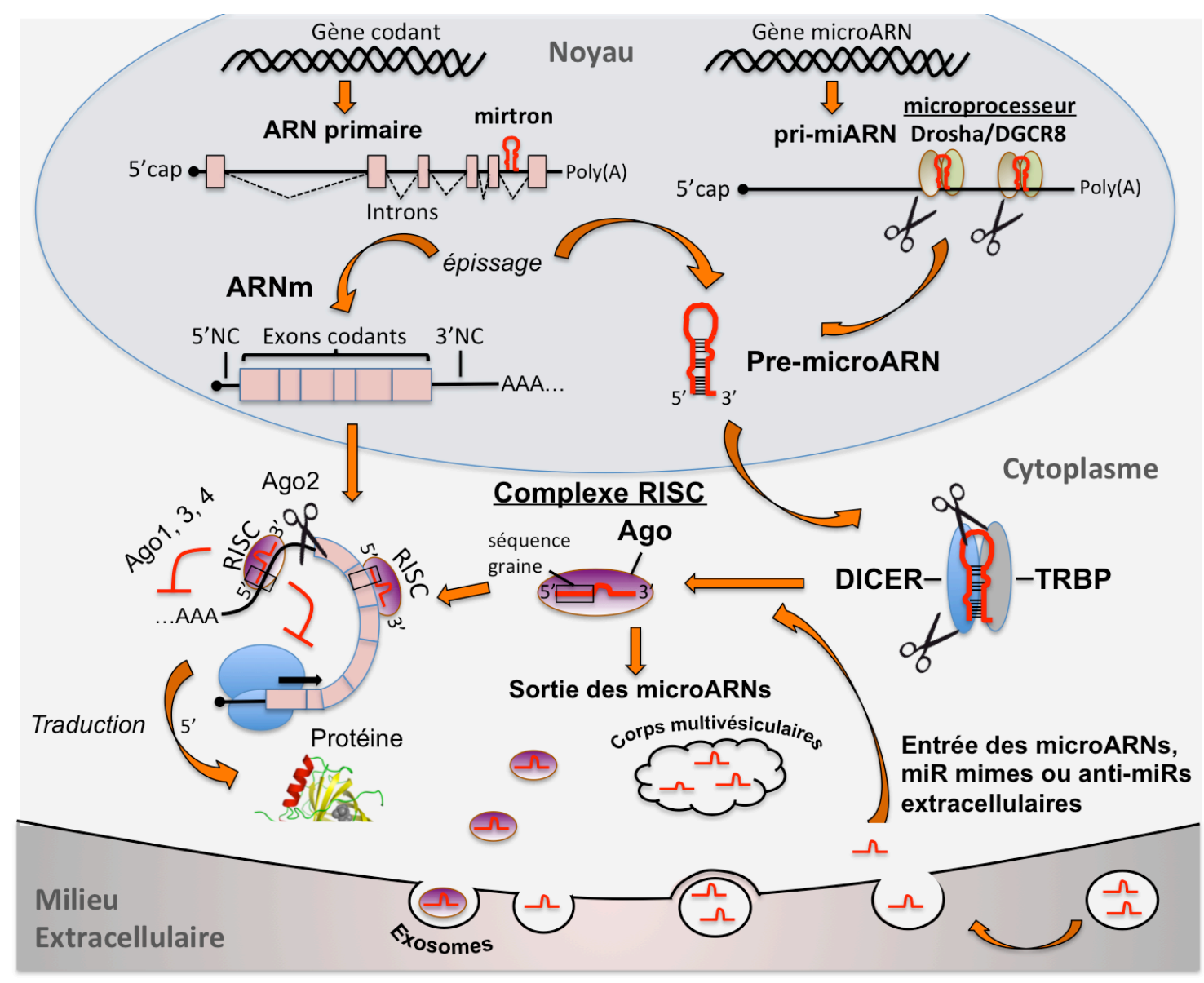


La Presse Médicale 2015: Journees Europeennes De La Societe Française De Cardiologie Dossier thématique : Les miARNs dans les pathologies cardiovasculaires Mathias Mericskay

Figure 2

A ARN primaire Ppargc1b codant PGC1 $\beta$ (PPARy coactivator $1 \beta$ )

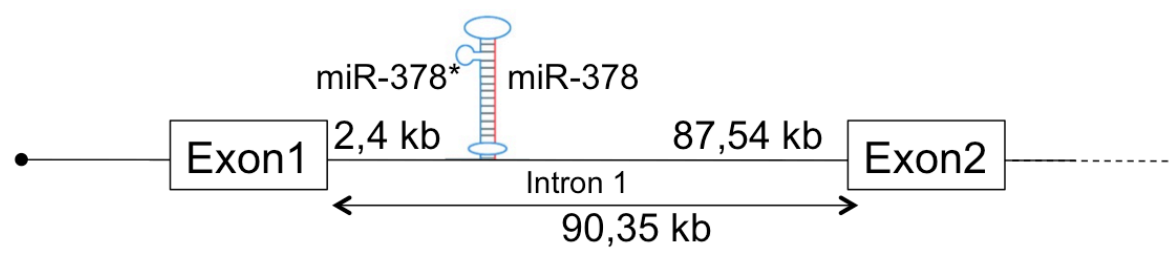

B Pre-miR

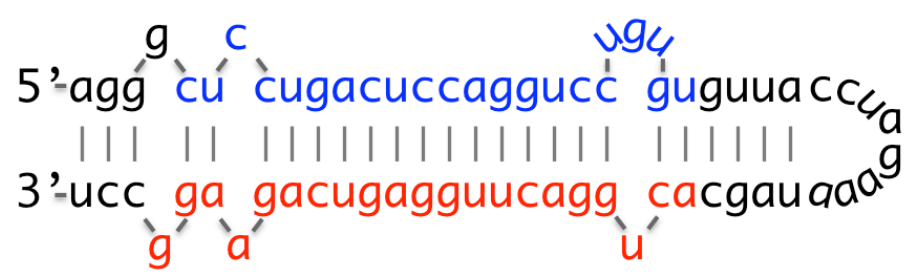

C miRs matures Séquence graine

miR-378-5p (*) 5' - cuccugdcuccagguccugugu-3'

miR-378-3p 5' -a cuggacuuggagucagaagg-3' 
La Presse Médicale 2015: Journees Europeennes De La Societe Française De Cardiologie Dossier thématique : Les miARNs dans les pathologies cardiovasculaires Mathias Mericskay

Figure 3
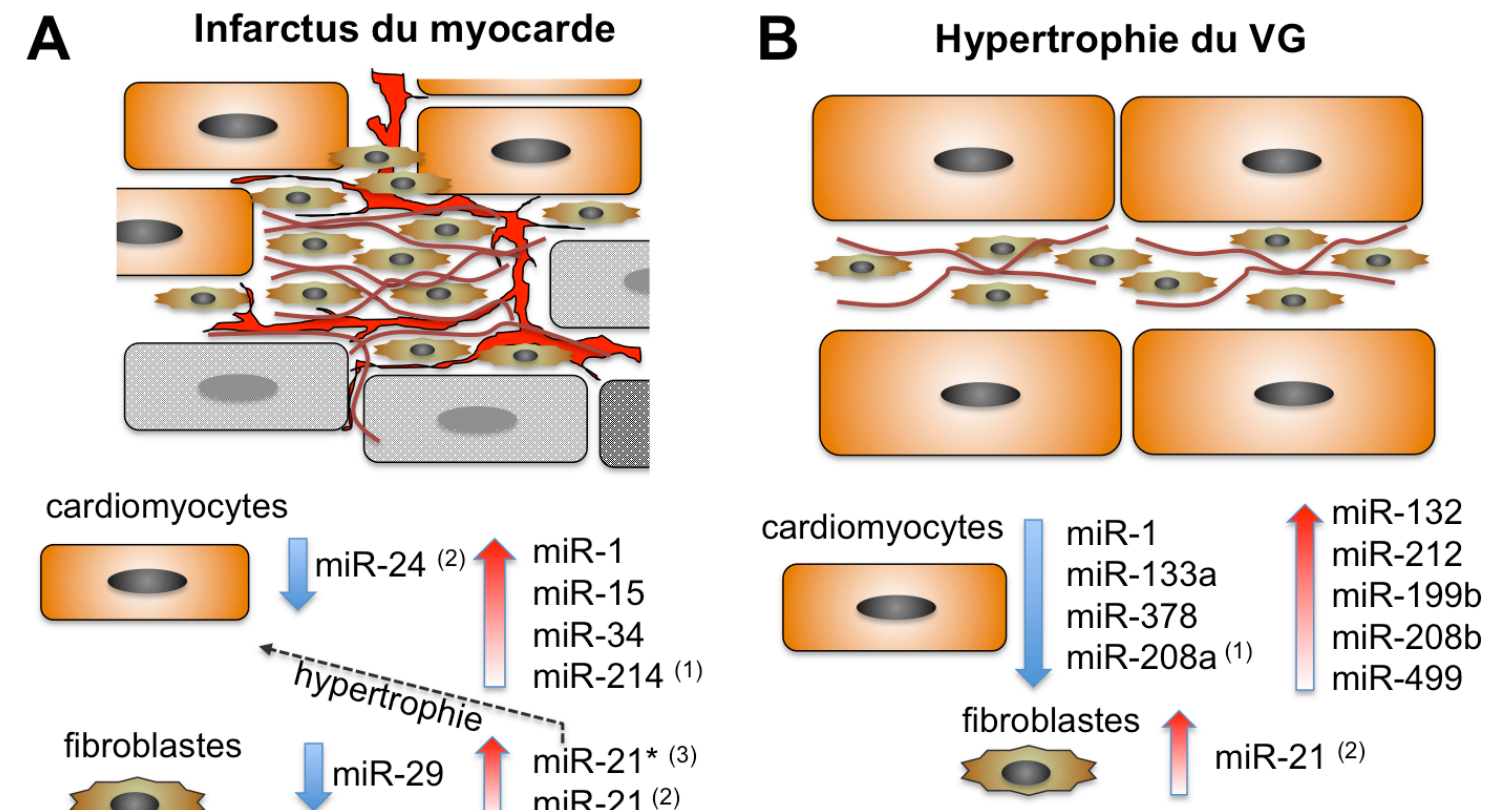

cardiomyocytes

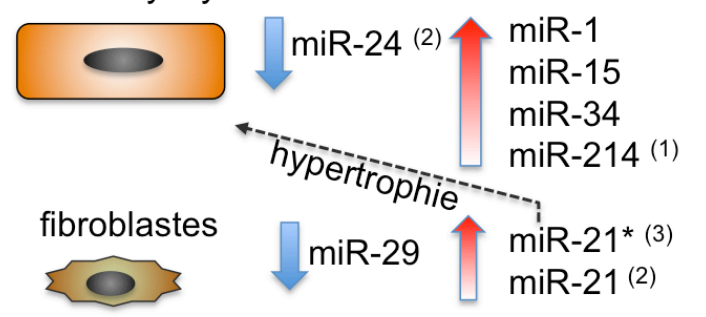

C Pathologies du muscle lisse artériel

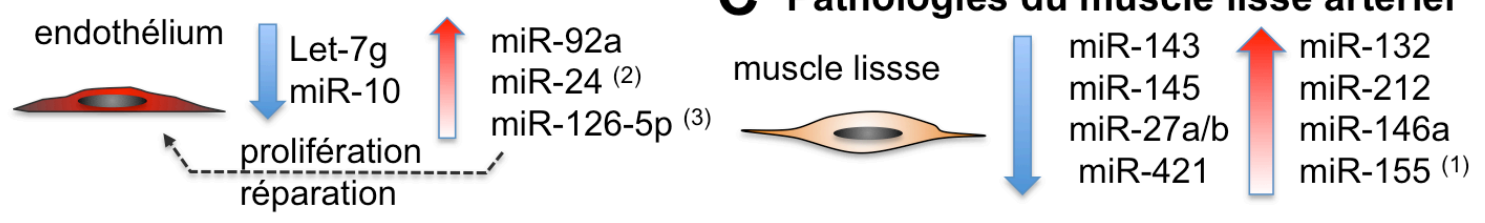


La Presse Médicale 2015: Journees Europeennes De La Societe Française De Cardiologie Dossier thématique : Les miARNs dans les pathologies cardiovasculaires Mathias Mericskay

Figure 4

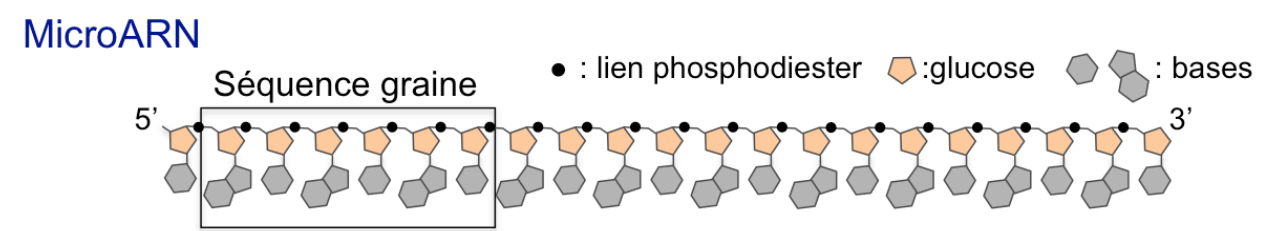

Antagomir

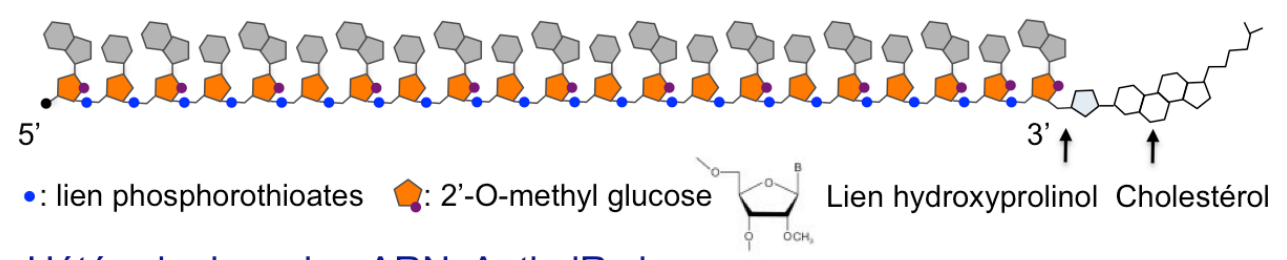

Hétéroduplex microARN: AntimiR clong

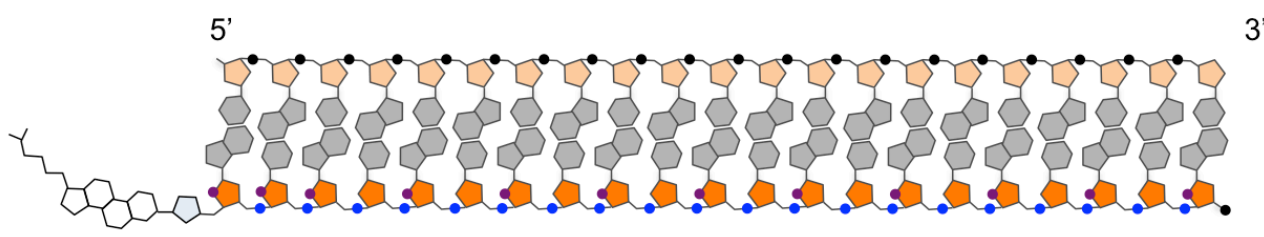

3'

5

Hétéroduplex microARN: AntimiR LNA court

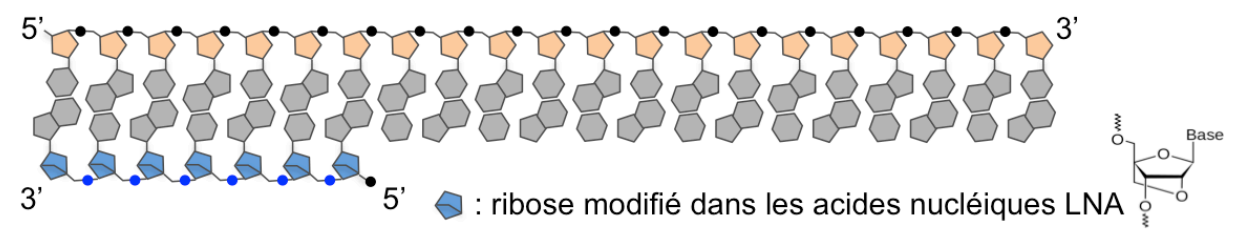

